\title{
The effectiveness of zinc in alleviating salinity stress on pistachio seedlings
}

\author{
Vahid Tavallali ${ }^{\star}$ \\ Department of Agriculture Payame Noor University (PNU), P.O. Box, 19395-3697 Tehran, Iran
}

Received 10 August 2015 - Accepted 7 July 2016

\begin{abstract}
Introduction. Zinc (Zn) deficiency is a common nutritional disorder in Pistachio (Pistacia vera) which is grown on calcareous, saline and sodic soils. The effects of $\mathrm{Zn}$ amendment on the mineral nutrient uptake, the osmoregulation and the dynamics of phytohormones in pistachio under salt stress were investigated in the common conditions of Iran Materials and methods. A greenhouse study was conducted to evaluate the improving effects of zinc $(0,5,10$ and $20 \mathrm{mg} \mathrm{Zn} \mathrm{kg}^{-1}$ soil) under saline (800, 1600, 2400 and $3200 \mathrm{mg} \mathrm{NaCl} \mathrm{kg}^{-1}$ soil) conditions on pistachio 'Badami' seedlings. The concentrations of potassium, calcium, sodium, magnesium, and $\mathrm{Zn}$ were determined by atomic absorption spectroscopy, and chloride was measured by titration Specific absorption rates (SAR) and specific utilization rates (SUR) of the minerals were calculated. Abscisic acid (ABA), 3-indoleacetic acid (IAA) and cytokinin contents were measured by HPLC, and the concentrations of osmoregulators (proline, glycine betaine and choline) were determined by spectrophotometry Results and discussion. The K, Ca and Zn concentrations and the IAA and cytokinin contents were reduced under Zn deficiency and salt stress. Increasing salinity in soil under Zn-deficient conditions generally decreased the SAR and SUR of K, Ca, Zn and Mg and ABA contents. However, these adverse effects of salinity were alleviated by increasing $\mathrm{Zn}$ levels up to $10 \mathrm{mg} \mathrm{kg}^{-1}$ soil. The addition of $\mathrm{Zn}$ to the soil significantly decreased the proline and choline concentrations and increased the glycine betaine one of the pistachio seedlings exposed to salinity. Zinc treatments influenced the relationship between the relative growth rate (RGR) and the studied elements SARs and SURs. Conclusion. Overall, Zn improved plant growth under salt stress. According to the results obtained in this study, adequate $\mathrm{Zn}$ treatments can prevent the uptake and accumulation of sodium in the pistachio leaf and stem and improve both plant RGR and mineral nutrients absorption. Adequate $\mathrm{Zn}$ treatments also contribute to maintain the balance among seedling phytohormones even under salinity stress.
\end{abstract}

Keywords: Iran / pistachio / Pistacia vera / osmoregulation / phytohormone / growth rate / specific absorption rate / specific utilization rate

Résumé - Efficacité du zinc contre le stress salin sur semis de pistachier. Introduction. La carence Zinc (Zn) est un trouble nutritionnel commun des pistachiers (Pistacia vera) cultivés en sols calcaires, salins et sodiques. Les effets d'une fertilisation en $\mathrm{Zn}$ sur l'absorption des nutriments minéraux, sur l'osmorégulation et sur la dynamique des phytohormones du pistachier soumis au stress salin ont été étudiés dans des conditions couramment rencontrées en Iran. Matériel et méthodes. L'étude a été menée sous serre pour évaluer les effets positifs du zinc ( 0,5 , 10 et $20 \mathrm{mg} \mathrm{Zn} \mathrm{kg}^{-1}$ de sol) sur la croissance des jeunes plants de pistachier cv. Badami en conditions de stress salin liées à une solution saline $\left(800,1600,2400 \mathrm{ou} 3200 \mathrm{mg} \mathrm{NaCl} \mathrm{kg}^{-1}\right.$ de sol). Les concentrations de potassium, calcium, sodium, magnésium et zinc ont été déterminées par spectroscopie d'absorption atomique, et le chlorure a été mesuré par titrage. Les taux spécifiques d'absorption (SAR) et les taux d'utilisation spécifiques (SUR) des minéraux ont été calculés. Les teneurs en acide abscissique (ABA), acide 3-indole acétique (IAA) et en cytokinines (CKs) ont été mesurées par HPLC, et les concentrations de divers régulateurs osmotiques (proline, glycine bétaïne et choline) ont été déterminées par spectrophotométrie. Résultats et discussion. Les concentrations en $\mathrm{K}$, Ca $\mathrm{Zn}$ et les teneurs en IAA et CKs ont diminué en conditions de carence en $\mathrm{Zn}$ et de stress salin. L'augmentation de la salinité dans un sol carencé en Zn a généralement diminué les SAR et SUR en $\mathrm{K}, \mathrm{Ca}, \mathrm{Zn}$ et $\mathrm{Mg}$ ainsi que la teneur

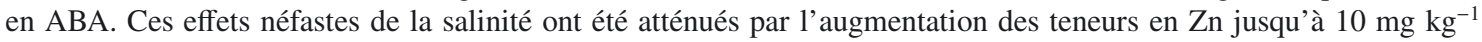
de sol. L'addition de $\mathrm{Zn}$ au sol a diminué de façon significative les teneurs en proline et en choline et a augmenté la concentration en glycine bétaïne des jeunes plants de pistachier soumis au stress salin. Les traitements au zinc ont influencé la relation entre le taux relatif de croissance (RGR) ainsi que les taux SAR et SUR des éléments étudiés.

\footnotetext{
^ Corresponding author: vtavallali@gmail.com
} 
Conclusion. Dans l'ensemble, le zinc a amélioré la croissance des plantes sous stress salin. Selon les résultats obtenus dans cette étude, des traitements adéquats au Zn peuvent empêcher l'absorption et l'accumulation de sodium dans les feuilles et les tiges du pistachier et améliorer à la fois la croissance des plantes et l'absorption des minéraux nutritifs. Des traitements adéquats au $\mathrm{Zn}$ contribuent également à maintenir l'équilibre entre phytohormones des plantules soumises au stress salin.

Mots clés : Iran / pistachier / Pistacia vera / osmorégulation / régulateur de croissance végétale / taux de croissance / taux d'absorption spécifique / taux d'utilisation spécifique

\section{Introduction}

Soil salinity is one of the major abiotic stresses that adversely affect plant productivity and quality in both irrigated and non-irrigated areas of the world [1], due mainly to an excess of $\mathrm{Cl}$ and $\mathrm{Na}^{+}$ions in plants [2]. Salinity stress limits plant development by adversely affecting various biochemical reactions and physiological processes such as photosynthesis, antioxidant metabolism, mineral nutrients homeostasis, osmolytes accumulation and hormonal signaling $[3,4]$. It is thought that the repressive effect of salinity on plant growth could be related to a decline in endogenous levels of phytohormones [5]. Furthermore high salt stress increases the deposition rate of $\mathrm{Na}$ in the growing zone of the root and hence decreases the selectivity for potassium $(\mathrm{K})$ versus $\mathrm{Na}$ [6]. Uptake of essential ions (both cations and anions) including $\mathrm{K}^{+}, \mathrm{Ca}^{+2}$, magnesium $\left(\mathrm{Mg}^{+2}\right)$, ammonium $\left(\mathrm{NH}^{4+}\right)$, and nitrate $\left(\mathrm{NO}^{3-}\right)$ have been reported to be suppressed in various pistachio cultivars by high levels of sodium chloride $(\mathrm{NaCl})$, especially in saline soil and irrigation water [7]. A large number of studies reported that the total nutrient uptake, accumulation, and nutrient partitioning within the plant were reduced by higher levels of salinity [8-10].

Poor quality of irrigation water in association with salt build-up soils has reduced the yields of pistachio (Pistacia vera L.) over recent years, especially in Kerman, and in central Iran, particularly in the Yazd and Qom regions. The symptoms of salinity in pistachio have been previously described $[8,10-12]$. For example, saline stress can decrease growth, alter gas exchange, enzyme activity, and protein metabolism, and cause morphological change in the leaves $[10,11,13]$.

Zinc deficiency is now recognized as one of the most critical micronutrient deficiency in plants grown on calcareous, saline and sodic soils with high $\mathrm{pH}$ values [14]. Zinc $(\mathrm{Zn})$ is an essential nutrient element for plants and plays a role in several plant physiological processes i.e., photosynthesis, respiration, and synthesis of protein, DNA, RNA, and plant hormones such as indole-3-acetic acid (IAA) metabolism [15]. Zinc is required for scavenging of reactive oxygen species (ROS) including superoxide radical $\left(\mathrm{O}_{2}^{-}\right)$and hydrogen peroxide $\left(\mathrm{H}_{2} \mathrm{O}_{2}\right)$ induced by salinity stress [16]. Zinc also alleviates the adverse effects of salinity on phytohormones levels [17]. Furthermore, $\mathrm{Zn}$ supply could mitigate the adverse effects of $\mathrm{NaCl}$ [18]. Welch et al. [19] stated that $\mathrm{Zn}$ is necessary for root cell membrane integrity. From this point of view, external $\mathrm{Zn}$ concentrations could mitigate the adverse effect of $\mathrm{NaCl}$ by inhibiting $\mathrm{Na}$ and/or $\mathrm{Cl}$ uptake or translocation. Alpaslan et al. [20] concluded that in the salt affected areas, Zn ap- plication could alleviate possible $\mathrm{Na}$ and $\mathrm{Cl}$ injury in plants. Fertilizer management can strongly affect plant growth and development under salinity condition by changing their nutritional status [21].

There is limited work dealing with the interaction of $\mathrm{Zn} /$ salinity on pistachio. This experiment was, therefore, conducted on 'Badami' rootstock which is an important Iranian fruit producing cultivar for evaluation of responses to $\mathrm{Zn}$ fertilization and salinity on uptake and distribution of some macro and micronutrients in plant organs and phytohormone and osmolyte contents.

\section{Materials and methods}

\subsection{Plant material and treatments}

The experiments were conducted from February 2014 to December 2014 at the Agricultural research greenhouse of Shiraz Payame Noor University. Soil used in these studies was a loam taken from 0 to $30 \mathrm{~cm}$ depth of Chitgar series soils (Fine-loamy, carbonatic, thermic Typic Calcixerepts) located at Sarvestan township, $85 \mathrm{~km}$ southeast of Shiraz. Some physical and chemical properties of the soil are given in table I. The soil samples air-dried, crushed to pass through a 2-mm sieve and $\mathrm{Zn}$ treatments were combined thoroughly with soil and supplied at the rate of $0,5,10$ and $20 \mathrm{mg} \mathrm{kg}^{-1}$ soil as $\mathrm{ZnSO}_{4} .7 \mathrm{H}_{2} \mathrm{O}$. Zinc treated soils were put in 8 -L plastic pots.

Pistachio (Pistacia vera L. cv. Badami) seeds were placed in muslin sacks and soaked for $24 \mathrm{~h}$ in $0.4 \%$ captan solution. Seeds were then planted in sand and kept at $30{ }^{\circ} \mathrm{C}$ for one week. Four germinated seeds were planted in each pot and all 80 pots were irrigated with deionized water twice a week to keep the soil water content higher than the field capacity (on 20\%, soil dry weight basis). Nitrogen and P at the rate of $50 \mathrm{mg} \mathrm{kg}^{-1}$ soil, and $\mathrm{Cu}$ and $\mathrm{Mn}$ at the rate of $5 \mathrm{mg} \mathrm{kg}^{-1}$ soil were applied uniformly to all pots each as $\mathrm{NH}_{4} \mathrm{NO}_{3}, \mathrm{KH}_{2} \mathrm{PO}_{4}$, $\mathrm{CuSO}_{4} .5 \mathrm{H}_{2} \mathrm{O}$ and $\mathrm{MnSO}_{4} \cdot \mathrm{H}_{2} \mathrm{O}$, respectively. After 25 days the 4-leaved seedlings were thinned to two uniform seedlings per pot. Seven days later, salt treatments $(0,800,1600,2400$ and $3200 \mathrm{mg} \mathrm{NaCl} \mathrm{kg}$ soil) were added to the pots at 3day intervals using $0.5 \mathrm{~L}$ irrigation water. After 50 and 100 days salt treatments, measurements were performed. Data are only shown for plants harvested after a 100-day salinization period. Twenty treatments were arranged in a factorial experiment based on completely randomized design (CRD) with 4 replications. 
Table I. Some physical and chemical properties of the soil used in the experiment.

\begin{tabular}{|c|c|c|c|c|c|c|c|c|}
\hline \multicolumn{9}{|c|}{ Water content (\%, dry weight basis) } \\
\hline Loam & 20 & 10 & 7.8 & 1.2 & 13.5 & 63 & 12 & 1.7 \\
\hline
\end{tabular}

\subsection{Elemental analysis}

Concentrations of different elements were determined in roots, stems, and the leaves of seedlings. Oven dried plant materials were ground to a fine powder. A mass of $0.5 \mathrm{~g}$ dry samples was ashed at $500{ }^{\circ} \mathrm{C}$ heat for $8 \mathrm{~h}$, and the ash was dissolved in $5 \mathrm{~mL} 2 \mathrm{~N}$ hydrochloric acid $(\mathrm{HCl})$. After digestion, the volume of the sample was made up to $100 \mathrm{~mL}$ with distilled deionized water [22]. The concentrations of $\mathrm{Na}$ and $\mathrm{K}$ were estimated by flame photometry (Model Jenway PFP7 ELE Instrument Co. Ltd.) and analyses of $\mathrm{Zn}, \mathrm{Ca}$ and $\mathrm{Mg}$ were carried out with an atomic absorption spectrometer (Model Varian 220, Australia). Chloride $\left(\mathrm{Cl}^{-}\right)$was extracted from $0.1 \mathrm{~g}$ of ground dried material with $50 \mathrm{~mL}$ of deionized water and measured by titration with silver nitrate [8]. The specific absorption rate, SAR $\left(\mathrm{mg} \mathrm{g}^{-1} \mathrm{day}^{-1}\right)$, an index of the element uptake efficiency of roots, was calculated using the formula

$$
S A R=1 / R D W \partial M / \partial T
$$

where $R D W$ is the root dry weight $(\mathrm{g}), M$ is the element amount (mg) in the whole plant and $T$ is the time of harvest in days. The specific utilization rate on a leaf basis, $\mathrm{SUR}_{\mathrm{L}}(\mathrm{g}$ $\mathrm{mg}^{-1}$ day $^{-1}$ ), an index of the efficiency of the element in producing biomass, was calculated as the rate of plant biomass production per unit of element in the leaves [23,24]. The relationships between relative growth rate and $S A R$ and $S_{L}$ for the two harvests 50 and 100 days after salt treatment were evaluated by using regression equations.

\subsection{Phytohormone quantification}

\subsubsection{Phytohormone extraction and purification}

Phytohormones were extracted and purified according to Novakova et al. [25]. Frozen leaf samples (about $1 \mathrm{~g} \mathrm{FW}$ ) were ground in liquid nitrogen and extracted overnight with $10 \mathrm{~cm}^{3}$ methanol/water/formic acid (15/4/1, by vol., $\mathrm{pH} \sim 2.5,-20{ }^{\circ} \mathrm{C}$ ). For analyses of endogenous cytokinins (CKs), $50 \mathrm{pmol}$ of each of the following 12 deuteriumlabelled standards were added: $\left[{ }^{2} \mathrm{H}_{5}\right] \mathrm{Z}$, $\left[{ }^{2} \mathrm{H}_{5}\right] \mathrm{ZR}, \quad\left[{ }^{2} \mathrm{H}_{5}\right] \mathrm{Z7G}, \quad\left[{ }^{2} \mathrm{H} 5\right] \mathrm{Z} 9 \mathrm{G}, \quad\left[{ }^{2} \mathrm{H}_{5}\right] \mathrm{ZOG},\left[{ }^{2} \mathrm{H}_{5}\right] \mathrm{ZROG}$, $\left[{ }^{2} \mathrm{H}_{6}\right] \mathrm{iP},\left[{ }^{2} \mathrm{H}_{6}\right] \mathrm{iPR},\left[{ }^{2} \mathrm{H}_{6}\right] \mathrm{iP} 7 \mathrm{G},\left[{ }^{2} \mathrm{H}_{6}\right] \mathrm{iP} 9 \mathrm{G},\left[{ }^{2} \mathrm{H}_{5}\right] \mathrm{DHZ},\left[{ }^{2} \mathrm{H}_{5}\right]$ DHZR (Apex Organics, Honington, UK). Tritiated internal standards were used for the determination of auxin $\left(3\left[5(n)-{ }^{3} \mathrm{H}\right]\right.$ 3 -indoleacetic acid, Amersham, UK, specific activity 74 GBq $\mathrm{mmol}^{-1}, 5 \times 10^{3} \mathrm{~Bq}$ ) and ABA (Amersham, UK, specific activity $\left.1.74 \mathrm{TBq} \mathrm{mmol}^{-1}, 5 \times 10^{3} \mathrm{~Bq}\right)$. The extracts were purified using Si-C 18 columns (SepPak Plus, Waters, Milford, MC, USA) and Oasis MCX mixed mode (cation exchange and reverse phase) columns (150 mg, Waters, USA) and evaporated.

\subsubsection{HPLC of auxin and abscisic acid}

The auxin (3-indolyeacetic acid, IAA) and abscisic acid (ABA) were determined using two-dimensional HPLC according to Dobrev et al. [26]. The instrumental set-up consisted of a series 200 autosampler (Perkin Elmer, Norwalk, CT, USA), two HPLC gradient pump systems (first pump: ConstaMetric 3500 and 3200 with 50011 mixer, TSP, Riviera Beach, FL, USA; second pump: Series 200 Quaternary Pump, Perkin Elmer), two columns (first column: ACE-3CN, $150 \times 4.6 \mathrm{~mm}$, $3 \mathrm{~mm}$, ACT, Aberdeen, Scotland, UK; second column: Luna C18(2), $150 \times 4.6 \mathrm{~mm}, 3 \mathrm{~mm}$, Phenomenex, Torrance, CA, USA), one 2-position, fluid processor SelectPRO with $1 \mathrm{ml}$ loop (Alltech, Deerfield, IL, USA). The segment containing IAA and ABA obtained in the first dimension was collected in the loop of the fluid processor and redirected to the second HPLC dimension. IAA was quantified using fluorescence detector LC 240 (Perkin Elmer). Quantification of ABA was performed on the basis of UV detection using diode array-detector 235C (Perkin Elmer).

\subsubsection{HPLC/mass spectrometry}

LC-MS analysis was performed as described by Lexa et al. [27] using a Rheos 2000 HPLC gradient pump (Flux Instruments, Switzerland) and HTS PAL autosampler (CTC Analytics, Switzerland) coupled to an Ion Trap Mass Spectrometer Finnigan MAT LCQ-MS ${ }^{\mathrm{n}}$ equipped with an electrospray interface. Detection and quantification were carried out using a Finnigan LCQ operated in the positive ion, full-scan MS/MS mode using a multilevel calibration graph with deuterated cytokinins as internal standards. The detection limit was calculated for each compound as $3.3 \sigma / \mathrm{S}$, where $\sigma$ is the standard deviation of the response and $\mathrm{S}$ the slope of the calibration curve. Each sample was injected at least twice.

\subsection{Quantification of proline}

Quantification of proline was performed using the described method by Bates et al. [28]. Proline sample from pistachio leaf was extracted with $3 \%(\mathrm{w} / \mathrm{v})$ sulfosalicylic acid, and the extractions was then added to the mixtures of ninhydrin reagent and glacial acetic acid and boiled at $100{ }^{\circ} \mathrm{C}$ for $30 \mathrm{~min}$. After cooling to room temperature, $4 \mathrm{~mL}$ toluene was added and stirred well for 20-30 s. Later, the toluene layer was separated and allowed to retain room temperature. The proline concentration in red phase was determined at $520 \mathrm{~nm}$ of absorbance and expressed as $\mu \mathrm{mol} \mathrm{g}^{-1} \mathrm{FW}$. 


\subsection{Choline and glycine betaine determination}

To begin with, for total quaternary ammonium compound determination, dried finely-ground pistachio leaf $(0.1 \mathrm{~g})$ was mechanically shaken with $4 \mathrm{~mL}$ of deionized $\mathrm{H}_{2} \mathrm{O}$ for $24 \mathrm{~h}$ at $25{ }^{\circ} \mathrm{C}$ Filtered extracts were diluted $1: 1$ with $2 \mathrm{~N} \mathrm{H}_{2} \mathrm{SO}_{4}$. Aliquots $(0.50 \mathrm{~mL})$ were measured into heavy-walled glass centrifuge tubes and cooled in ice water for $1 \mathrm{~h}$. Cold $\mathrm{KI}-\mathrm{I}_{2}$ reagent $(0.20 \mathrm{~mL})$, prepared by dissolving $15 \mathrm{~g}$ of iodine and $2.0 \mathrm{~g}$ of $\mathrm{KI}$ in $100 \mathrm{~mL}$ water was added and the reactants were gently stirred with a vortex mixer. The tubes were stored at 0 $4{ }^{\circ} \mathrm{C}$ for $16 \mathrm{~h}$ and then centrifuged at $10000 \mathrm{rpm}$ for $15 \mathrm{~min}$ at $0{ }^{\circ} \mathrm{C}$. The tubes were kept in ice and the supernatant which contained the periodide complex was carefully aspirated with a fine tipped glass tube. The periodide crystals were dissolved in $9.0 \mathrm{~mL}$ of 1,2-dichloroethane (reagent grade). Vigorous vortex mixing was frequently required to affect complete solution in the developing solvent. After 2.0-2.5 h, the absorbance was measured at $365 \mathrm{~nm}$ with a Jenway Spectrophotometer model 7305 Reference standards of glycine betaine (GB) (50$200 \mu \mathrm{g} \mathrm{mL}^{-1}$ ) were prepared in $1 \mathrm{~N} \mathrm{H}_{2} \mathrm{SO}_{4}$ [29].

For choline determination, sample extracts were diluted $1: 1$ with KPi buffer $(0.2 \mathrm{M}, \mathrm{pH} 6.8)$. The choline periodides were precipitated and analyzed as previously described for total QAC. Glycine betaineperiodides do not precipitate at this pH.Total QAC, minus the concentration of choline, gave GB levels. The contents were expressed in $\mu \mathrm{g} \mathrm{g}^{-1}$ DW [29].

\subsection{Statistical analysis}

Data were presented as the means for each treatment $(n=$ 4). Data were subjected to analysis of variance (ANOVA) and means were compared using the Tukey's honest significant difference (HSD) test at the 5\% probability level. Analysis of variance was performed using the SPSS software (ver. 22.0) SPSS Inc.

\section{Results and discussion}

Zinc (Zn) is an essential nutrient element for higher plants and is mainly absorbed in the form of $\mathrm{Zn}^{++}$. It plays an important role in several plant physiological process i.e., photosynthesis, respiration, and synthesis of protein, DNA, RNA, and plant hormones [21]. Zn also plays other indirect and significant roles as stabilizer of proteins, membranes, and DNAbinding proteins such as $\mathrm{Zn}$-fingers [20] deficiency of $\mathrm{Zn}$ is one of the most common micronutrient deficiencies in plants worldwide [16]. The critical value of $\mathrm{Zn}$ deficiency for pistachios was determined to be about $7.0 \mathrm{mg} \mathrm{kg}^{-1}$ dry matter, while the adequate amount is determined to be about $1015 \mathrm{mg}$ $\mathrm{kg}^{-1}$ dry matter [31,32].

In the current study, salinity treatments led to a significant decrease in $\mathrm{Zn}$ concentration of leaf, stem and root tissues, however no such constant trend was found with increasing $\mathrm{NaCl}$ concentration. Zinc concentrations of the leaf, stem and stem tissues were lower at no $\mathrm{Zn}$ application treatments. At each salt treatment, increasing $\mathrm{Zn}$ accordingly increased $\mathrm{Zn}$ concentration of the seedling tissues (table II).
Table II. Effects of zinc ( $\mathrm{Zn}$ ) treatments on the $\mathrm{Zn}$ content of leaves, stems and roots of Pistacia vera $\mathrm{L}$. cv. Badami seedlings under $\mathrm{NaCl}$ stress (DW: dry weight). Values are means of 4 replicates.

\begin{tabular}{|c|c|c|c|c|c|}
\hline \multicolumn{6}{|c|}{ Zn levels $\left(\mathrm{mg} \mathrm{kg}^{-1}\right)$} \\
\hline $\mathrm{NaCl}$ levels ( $\mathrm{mg} \mathrm{kg}^{-1}$ ) & 0 & 5 & 10 & 20 & Means \\
\hline \multicolumn{6}{|c|}{ Leaf $\mathrm{Zn}\left(\mathrm{mg} \mathrm{kg}^{-1} \mathrm{DW}\right)$} \\
\hline 0 & $8.8 \mathrm{efg}^{\dagger}$ & 9.1 efg & $10.8 \mathrm{de}$ & $16.9 \mathrm{~b}$ & $11.4 \mathrm{~B}$ \\
\hline 800 & $8.0 \mathrm{fg}$ & $8.0 \mathrm{fg}$ & $10.0 \mathrm{ef}$ & $10.8 \mathrm{de}$ & $9.2 \mathrm{D}$ \\
\hline 1,600 & $4.5 \mathrm{j}$ & $7.7 \mathrm{gh}$ & $9.8 \mathrm{ef}$ & $12.5 \mathrm{~d}$ & $8.6 \mathrm{D}$ \\
\hline 2,400 & $5.5 \mathrm{ij}$ & $9.5 \mathrm{efg}$ & $12.7 \mathrm{~cd}$ & $21.9 \mathrm{a}$ & $12.4 \mathrm{~A}$ \\
\hline 3,200 & 5.7 hij & 7.5 ghi & $12.8 \mathrm{~cd}$ & $14.7 \mathrm{c}$ & $10.1 \mathrm{C}$ \\
\hline Mean & $6.5 \mathrm{D}$ & $8.3 \mathrm{C}$ & $11.2 \mathrm{~B}$ & $15.3 \mathrm{~A}$ & \\
\hline \multicolumn{6}{|c|}{ Stem $\mathrm{Zn}\left(\mathrm{mg} \mathrm{kg}^{-1} \mathrm{DW}\right)$} \\
\hline 0 & $8.5 \mathrm{~h}$ & $9.9 \mathrm{gh}$ & $16.9 \mathrm{f}$ & $30.3 \mathrm{a}$ & $16.4 \mathrm{~B}$ \\
\hline 800 & $4.4 \mathrm{i}$ & $9.6 \mathrm{gh}$ & $11.4 \mathrm{~g}$ & $16.9 \mathrm{f}$ & $10.5 \mathrm{D}$ \\
\hline 1,600 & $8.7 \mathrm{~h}$ & $11.0 \mathrm{~g}$ & $17.8 \mathrm{f}$ & $26.5 \mathrm{~b}$ & $16.0 \mathrm{BC}$ \\
\hline 2,400 & $5.4 \mathrm{i}$ & $17.7 \mathrm{f}$ & 18.4 ef & $20.3 \mathrm{de}$ & $15.4 \mathrm{C}$ \\
\hline 3,200 & $10.0 \mathrm{gh}$ & $20.5 \mathrm{~d}$ & $23.1 \mathrm{c}$ & $29.6 \mathrm{a}$ & $20.8 \mathrm{~A}$ \\
\hline Mean & $7.4 \mathrm{D}$ & $13.7 \mathrm{C}$ & $17.5 \mathrm{~B}$ & $24.7 \mathrm{~A}$ & \\
\hline \multicolumn{6}{|c|}{ Root $\mathrm{Zn}\left(\mathrm{mg} \mathrm{kg}^{-1} \mathrm{DW}\right)$} \\
\hline 0 & $7.0 \mathrm{k}$ & 15.9 ef & $22.4 \mathrm{c}$ & $26.3 \mathrm{~b}$ & $17.9 \mathrm{~B}$ \\
\hline 800 & $12.1 \mathrm{hij}$ & $12.9 \mathrm{ghi}$ & $16.5 \mathrm{e}$ & $19.0 \mathrm{~d}$ & 15.1 D \\
\hline 1,600 & $10.6 \mathrm{j}$ & $10.8 \mathrm{j}$ & $16.3 \mathrm{ef}$ & $29.1 \mathrm{a}$ & $16.7 \mathrm{C}$ \\
\hline 2,400 & $6.1 \mathrm{k}$ & $13.5 \mathrm{gh}$ & $14.3 \mathrm{fg}$ & $24.0 \mathrm{c}$ & $14.4 \mathrm{D}$ \\
\hline 3,200 & $11.0 \mathrm{ij}$ & 15.7 ef & $23.1 \mathrm{c}$ & $26.1 \mathrm{~b}$ & $18.9 \mathrm{~A}$ \\
\hline \multirow[t]{2}{*}{ Mean } & $9.3 \mathrm{D}$ & $13.7 \mathrm{C}$ & $18.5 \mathrm{~B}$ & $24.9 \mathrm{~A}$ & \\
\hline & Leaf $\mathrm{Zn}$ & \multicolumn{2}{|c|}{ Stem Zn } & \multicolumn{2}{|c|}{ Root Zn } \\
\hline $\mathrm{NaCl}$ level & $* *$ & \multicolumn{2}{|c|}{$* *$} & \multicolumn{2}{|c|}{$* *$} \\
\hline Zn level & $* *$ & \multicolumn{2}{|c|}{$* *$} & \multicolumn{2}{|c|}{$* *$} \\
\hline $\mathrm{NaCl} \times \mathrm{Zn}$ & $* *$ & \multicolumn{2}{|c|}{$* *$} & \multicolumn{2}{|c|}{$* *$} \\
\hline
\end{tabular}

** Significant at $P \leq 0.01$.

$\dagger$ Means followed by the same letter (small letters for means and capital letters for means of main effects) are not significantly different according to Tukey's HSD test at $P \leq 0.05$.

Previous studies also reported the uptake and utilization of $\mathrm{Zn}$ as well as the absorption and utilization of $\mathrm{K}$ and $\mathrm{Ca}$ in wheat, rice, and pepper seedlings decreased with elevated soil salinity [32-34]. The relatively high concentrations of $\mathrm{Na}$ and/or limited water availability to plants caused by excess soluble salts were probably responsible for the decrease in mineral nutrients concentrations in tissues under saline condition. Genc et al. [35] stated that harmful effects of $\mathrm{Zn}$ deficiency under $\mathrm{NaCl}$ stress may act as a greater limiting factor than $\mathrm{NaCl}$ toxicity in reducing growth. 
Table III. Effects of zinc ( $\mathrm{Zn}$ ) treatments on the Na content of leaves, stems and roots of Pistacia vera $\mathrm{L}$. cv. Badami seedlings under $\mathrm{NaCl}$ stress (DW: dry weight). Values are means of 4 replicates.

\begin{tabular}{|c|c|c|c|c|c|}
\hline \multicolumn{6}{|c|}{$\mathrm{Zn}$ levels $\left(\mathrm{mg} \mathrm{kg}^{-1}\right)$} \\
\hline $\begin{array}{l}\mathrm{NaCl} \text { levels } \\
\left(\mathrm{mg} \mathrm{kg}^{-1}\right)\end{array}$ & 0 & 5 & 10 & 20 & Means \\
\hline \multicolumn{6}{|c|}{ Leaf $\mathrm{Na}\left(\mathrm{mg} \mathrm{kg}^{-1} \mathrm{DW}\right)$} \\
\hline 0 & $1.10 \mathrm{gh}^{\dagger}$ & $0.60 \mathrm{i}$ & $0.60 \mathrm{i}$ & $0.60 \mathrm{i}$ & $0.72 \mathrm{E}$ \\
\hline 800 & $2.00 \mathrm{e}$ & $1.40 \mathrm{fg}$ & $0.60 \mathrm{i}$ & $0.60 \mathrm{i}$ & $1.15 \mathrm{D}$ \\
\hline 1,600 & $1.60 \mathrm{ef}$ & $1.40 \mathrm{fg}$ & $4.00 \mathrm{~d}$ & $2.00 \mathrm{e}$ & $2.25 \mathrm{C}$ \\
\hline 2,400 & $1.80 \mathrm{ef}$ & 0.90 hi & $4.20 \mathrm{~d}$ & $3.80 \mathrm{~d}$ & $2.67 \mathrm{~B}$ \\
\hline 3,200 & $12.70 \mathrm{a}$ & $8.00 \mathrm{~b}$ & $0.70 \mathrm{hi}$ & $5.20 \mathrm{e}$ & $6.65 \mathrm{~A}$ \\
\hline Mean & $3.84 \mathrm{~A}$ & $2.46 \mathrm{~B}$ & $2.02 \mathrm{C}$ & $2.44 \mathrm{~B}$ & \\
\hline \multicolumn{6}{|c|}{ Stem Na $\left(\mathrm{g} \mathrm{kg}^{-1} \mathrm{DW}\right)$} \\
\hline 0 & $0.50 \mathrm{i}$ & $0.70 \mathrm{hi}$ & $0.90 \mathrm{fg}$ & $0.60 \mathrm{hi}$ & $0.67 \mathrm{D}$ \\
\hline 800 & $0.60 \mathrm{hi}$ & 0.60 hi & 0.60 hi & $2.60 \mathrm{~b}$ & $1.10 \mathrm{C}$ \\
\hline 1,600 & $1.30 \mathrm{de}$ & $4.00 \mathrm{a}$ & $1.10 \mathrm{ef}$ & $0.90 \mathrm{fg}$ & $1.82 \mathrm{~B}$ \\
\hline 2,400 & $2.60 \mathrm{~b}$ & $1.40 \mathrm{~cd}$ & $1.40 \mathrm{~cd}$ & $1.60 \mathrm{c}$ & $1.75 \mathrm{~B}$ \\
\hline 3,200 & $3.80 \mathrm{a}$ & $1.60 \mathrm{c}$ & $2.80 \mathrm{~b}$ & $0.80 \mathrm{gh}$ & $2.25 \mathrm{~A}$ \\
\hline Mean & $1.76 \mathrm{~A}$ & $1.66 \mathrm{~B}$ & $1.36 \mathrm{C}$ & $1.30 \mathrm{C}$ & \\
\hline \multicolumn{6}{|c|}{ Root $\mathrm{Na}\left(\mathrm{g} \mathrm{kg}^{-1} \mathrm{DW}\right)$} \\
\hline 0 & $1.10 \mathrm{i}$ & $2.20 \mathrm{gh}$ & $3.20 \mathrm{e}$ & $2.40 \mathrm{~g}$ & $2.22 \mathrm{C}$ \\
\hline 800 & $2.20 \mathrm{gh}$ & $0.70 \mathrm{j}$ & $2.00 \mathrm{~h}$ & $2.40 \mathrm{~g}$ & $1.82 \mathrm{D}$ \\
\hline 1,600 & $2.80 \mathrm{f}$ & $4.50 \mathrm{~b}$ & $4.20 \mathrm{c}$ & $3.20 \mathrm{e}$ & $3.67 \mathrm{~B}$ \\
\hline 2,400 & $2.40 \mathrm{~g}$ & $4.50 \mathrm{~b}$ & $4.00 \mathrm{c}$ & $3.50 \mathrm{~d}$ & $3.60 \mathrm{~B}$ \\
\hline 3,200 & $0.50 \mathrm{j}$ & $4.50 \mathrm{~b}$ & $2.80 \mathrm{f}$ & $10.40 \mathrm{a}$ & $4.55 \mathrm{~A}$ \\
\hline \multirow[t]{2}{*}{ Mean } & $1.80 \mathrm{C}$ & $3.28 \mathrm{~B}$ & $3.24 \mathrm{~B}$ & $4.38 \mathrm{~A}$ & \\
\hline & Leaf $\mathrm{Na}$ & \multicolumn{2}{|c|}{ Stem Na } & \multicolumn{2}{|c|}{ Root Na } \\
\hline $\mathrm{NaCl}$ level & $* *$ & \multicolumn{2}{|c|}{$* *$} & \multicolumn{2}{|c|}{$* *$} \\
\hline Zn level & $* *$ & \multicolumn{2}{|c|}{$* *$} & \multicolumn{2}{|c|}{$* *$} \\
\hline $\mathrm{NaCl} \times \mathrm{Zn}$ & $* *$ & \multicolumn{2}{|c|}{$* *$} & \multicolumn{2}{|c|}{$* *$} \\
\hline
\end{tabular}

** Significant at $P \leq 0.01$.

$\dagger$ Means followed by the same letter (small letters for means and capital letters for means of main effects) are not significantly different according to Tukey's HSD test at $P \leq 0.05$.

Furthermore, in the current study showed that leaf, stem and root concentrations of sodium $(\mathrm{Na})$ increased with rising $\mathrm{NaCl}$ levels at each $\mathrm{Zn}$ treatment (table III). Application of $\mathrm{Zn}$ compared with control, decreased the $\mathrm{Na}$ concentration of leaf and stem of pistachio seedlings. However, at all salinity levels and control, plants supplied with $\mathrm{Zn}$ accumulated higher $\mathrm{Na}$ in root compared with the control (without $\mathrm{Zn}$ ). Presented results in table III clearly show that in root, $\mathrm{Na}$ concentration was higher than in leaf and stem. In addition, chloride $(\mathrm{Cl})$ concentration
Table IV. Effects of zinc ( $\mathrm{Zn}$ ) treatments on the $\mathrm{Cl}$ content of leaves, stems and roots of Pistacia vera $\mathrm{L}$. cv. Badami seedlings under $\mathrm{NaCl}$ stress (DW: dry weight). Values are means of 4 replicates.

\begin{tabular}{|c|c|c|c|c|c|}
\hline \multicolumn{6}{|c|}{ Zn levels $\left(\mathrm{mg} \mathrm{kg}^{-1}\right)$} \\
\hline $\begin{array}{l}\mathrm{NaCl} \text { levels } \\
\left(\mathrm{mg} \mathrm{kg}^{-1}\right)\end{array}$ & 0 & 5 & 10 & 20 & Means \\
\hline \multicolumn{6}{|c|}{ Leaf CI (mg kg$\left.{ }^{-1} \mathrm{DW}\right)$} \\
\hline 0 & $17.001^{\dagger}$ & $15.00 \mathrm{~m}$ & $6.75 \mathrm{o}$ & $4.00 \mathrm{p}$ & $10.68 \mathrm{E}$ \\
\hline 800 & $20.20 \mathrm{j}$ & 16.501 & $14.50 \mathrm{~m}$ & $12.50 \mathrm{n}$ & $15.92 \mathrm{D}$ \\
\hline 1,600 & $26.75 \mathrm{f}$ & $25.75 \mathrm{~g}$ & $22.75 \mathrm{~h}$ & $18.00 \mathrm{k}$ & $23.31 \mathrm{C}$ \\
\hline 2,400 & $31.00 \mathrm{~d}$ & $29.00 \mathrm{e}$ & $27.00 \mathrm{f}$ & $23.00 \mathrm{~h}$ & $27.50 \mathrm{~B}$ \\
\hline 3,200 & $38.75 \mathrm{a}$ & $37.00 \mathrm{~b}$ & $36.25 \mathrm{c}$ & $21.25 \mathrm{i}$ & $33.31 \mathrm{~A}$ \\
\hline Mean & $26.74 \mathrm{~A}$ & $24.65 \mathrm{~B}$ & $21.45 \mathrm{C}$ & $15.75 \mathrm{D}$ & \\
\hline \multicolumn{6}{|c|}{ Stem Cl $\left(\mathrm{g} \mathrm{kg}^{-1} \mathrm{DW}\right)$} \\
\hline 0 & 5.751 & $5.00 \mathrm{~m}$ & $4.00 \mathrm{n}$ & $3.25 \mathrm{o}$ & $4.50 \mathrm{D}$ \\
\hline 800 & $14.75 \mathrm{~b}$ & $6.25 \mathrm{jkl}$ & $4.50 \mathrm{mn}$ & $3.25 \mathrm{o}$ & $7.18 \mathrm{C}$ \\
\hline 1,600 & $9.50 \mathrm{c}$ & $8.00 \mathrm{e}$ & $6.25 \mathrm{jkl}$ & $6.00 \mathrm{kl}$ & $7.43 \mathrm{~B}$ \\
\hline 2,400 & $8.75 \mathrm{~d}$ & 7.50 efg & $6.75 \mathrm{hij}$ & $6.50 \mathrm{ijk}$ & $7.37 \mathrm{BC}$ \\
\hline 3,200 & $26.25 \mathrm{a}$ & 7.75 ef & $7.25 \mathrm{fgh}$ & 7.00 ghi & $12.06 \mathrm{~A}$ \\
\hline Mean & $13.00 \mathrm{~A}$ & $6.90 \mathrm{~B}$ & $5.75 \mathrm{C}$ & $5.20 \mathrm{D}$ & \\
\hline \multicolumn{6}{|c|}{ Root $\mathrm{Cl}\left(\mathrm{g} \mathrm{kg}^{-1} \mathrm{DW}\right)$} \\
\hline 0 & 8.75 ef & 8.50 efg & $7.75 \mathrm{~h}$ & $4.25 \mathrm{k}$ & $7.31 \mathrm{E}$ \\
\hline 800 & $9.50 \mathrm{~cd}$ & $9.00 \mathrm{de}$ & $8.25 \mathrm{fgh}$ & $6.25 \mathrm{j}$ & $8.25 \mathrm{D}$ \\
\hline 1,600 & $12.00 \mathrm{~b}$ & 8.75 ef & $8.00 \mathrm{gh}$ & $6.75 \mathrm{ij}$ & $8.87 \mathrm{C}$ \\
\hline 2,400 & $11.50 \mathrm{~b}$ & $10.00 \mathrm{c}$ & $9.75 \mathrm{c}$ & $9.50 \mathrm{~cd}$ & $10.18 \mathrm{~B}$ \\
\hline 3,200 & $14.50 \mathrm{a}$ & $11.50 \mathrm{~b}$ & $9.75 \mathrm{c}$ & $7.00 \mathrm{i}$ & $10.68 \mathrm{~A}$ \\
\hline \multirow[t]{2}{*}{ Mean } & $11.25 \mathrm{~A}$ & $9.55 \mathrm{~B}$ & $8.70 \mathrm{C}$ & $6.75 \mathrm{D}$ & \\
\hline & Leaf $\mathrm{Cl}$ & \multicolumn{2}{|c|}{ Stem Cl } & \multicolumn{2}{|c|}{ Root $\mathrm{Cl}$} \\
\hline $\mathrm{NaCl}$ level & $* *$ & \multicolumn{2}{|c|}{$* *$} & \multicolumn{2}{|c|}{$* *$} \\
\hline Zn level & $* *$ & \multicolumn{2}{|c|}{$* *$} & \multicolumn{2}{|c|}{$* *$} \\
\hline $\mathrm{NaCl} \times \mathrm{Zn}$ & $* *$ & \multicolumn{2}{|c|}{$* *$} & \multicolumn{2}{|c|}{$* *$} \\
\hline
\end{tabular}

** Significant at $P \leq 0.01$.

${ }^{\dagger}$ Means followed by the same letter (small letters for means and capital letters for means of main effects) are not significantly different according to Tukey's HSD test at $P \leq 0.05$.

of leaf, stem and root of pistachio seedlings was significantly increased under salinity stress. Zinc application markedly decreased $\mathrm{Cl}$ concentration of leaf, stem and root, at levels of 20, 10 and $5 \mathrm{mg} \mathrm{kg}^{-1}$ soil, respectively. Results showed that under $\mathrm{NaCl}$ treatments, decreasing effect of $\mathrm{Zn}$ application at level of $20 \mathrm{mg} \mathrm{kg}^{-1}$ soil on $\mathrm{Cl}$ concentration was approximately $41 \%$ in leaf, $60 \%$ in stem and $40 \%$ in root tissues (table IV). The increase in $\mathrm{Na}$ and $\mathrm{Cl}$ uptake and accumulation in plant tissues causes cell damages and death, and the increases were 
Table V. Effects of zinc ( $\mathrm{Zn}$ ) treatments on the Ca content of leaves, stems and roots of Pistacia vera $\mathrm{L}$. cv. Badami seedlings under $\mathrm{NaCl}$ stress (DW: dry weight). Values are means of 4 replicates.

\begin{tabular}{|c|c|c|c|c|c|}
\hline \multicolumn{6}{|c|}{$\mathrm{Zn}$ levels $\left(\mathrm{mg} \mathrm{kg}^{-1}\right)$} \\
\hline $\mathrm{NaCl}$ levels & 0 & 5 & 10 & 20 & Means \\
\hline$\left(\mathrm{mg} \mathrm{kg}^{-1}\right)$ & & & & & \\
\hline \multicolumn{6}{|c|}{ Leaf $\mathrm{Ca}\left(\mathrm{mg} \mathrm{kg}^{-1} \mathrm{DW}\right)$} \\
\hline 0 & $5.53 \mathrm{ab}^{\dagger}$ & $4.43 \mathrm{f}$ & $5.32 \mathrm{a}-\mathrm{d}$ & $5.29 \mathrm{a}-\mathrm{d}$ & $5.14 \mathrm{~B}$ \\
\hline 800 & $5.43 \mathrm{abc}$ & $5.75 \mathrm{ab}$ & $5.22 \mathrm{~b}-\mathrm{e}$ & $5.34 \mathrm{a}-\mathrm{d}$ & $5.43 \mathrm{Ab}$ \\
\hline 1,600 & $4.84 \mathrm{def}$ & $5.87 \mathrm{a}$ & $5.51 \mathrm{ab}$ & $5.38 \mathrm{a}-\mathrm{d}$ & $5.39 \mathrm{~A}$ \\
\hline 2,400 & $4.66 \mathrm{ef}$ & $5.57 \mathrm{ab}$ & $5.57 \mathrm{ab}$ & $4.87 \mathrm{c}-\mathrm{f}$ & $5.16 \mathrm{~B}$ \\
\hline 3,200 & $4.40 \mathrm{f}$ & $5.74 \mathrm{ab}$ & $5.43 \mathrm{abc}$ & $4.86 \mathrm{c}-\mathrm{f}$ & $5.10 \mathrm{~B}$ \\
\hline Mean & $4.96 \mathrm{~B}$ & $5.47 \mathrm{~A}$ & $5.41 \mathrm{~A}$ & $5.14 \mathrm{~B}$ & \\
\hline \multicolumn{6}{|c|}{ Stem Ca $\left(\mathrm{g} \mathrm{kg}^{-1} \mathrm{DW}\right)$} \\
\hline 0 & $3.80 \mathrm{~g}$ & $5.56 \mathrm{a}$ & $4.62 \mathrm{~b}-\mathrm{e}$ & $4.60 \mathrm{~b}-\mathrm{e}$ & $4.64 \mathrm{~B}$ \\
\hline 800 & $4.27 \mathrm{~d}-\mathrm{g}$ & $3.95 \mathrm{fg}$ & 4.22 efg & $5.01 \mathrm{ab}$ & $4.36 \mathrm{C}$ \\
\hline 1,600 & $4.15 \mathrm{efg}$ & $4.68 \mathrm{~b}-\mathrm{e}$ & $4.69 \mathrm{~b}-\mathrm{e}$ & $4.46 \mathrm{~b}-\mathrm{f}$ & $4.49 \mathrm{BC}$ \\
\hline 2,400 & 4.14 efg & $5.50 \mathrm{a}$ & $4.60 \mathrm{~b}-\mathrm{e}$ & $4.62 \mathrm{~b}-\mathrm{e}$ & $4.71 \mathrm{AB}$ \\
\hline 3,200 & $4.32 \mathrm{c}-\mathrm{g}$ & $5.49 \mathrm{a}$ & $4.86 \mathrm{bcd}$ & $4.88 \mathrm{bc}$ & $4.88 \mathrm{~A}$ \\
\hline Mean & $4.13 \mathrm{C}$ & $5.03 \mathrm{~A}$ & $4.59 \mathrm{~B}$ & $4.71 \mathrm{~B}$ & \\
\hline \multicolumn{6}{|c|}{ Root Ca $\left(\mathrm{g} \mathrm{kg}^{-1} \mathrm{DW}\right)$} \\
\hline 0 & $4.22 \mathrm{e}$ & $5.50 \mathrm{a}$ & $5.00 \mathrm{abc}$ & $4.87 \mathrm{bc}$ & $4.89 \mathrm{~A}$ \\
\hline 800 & $4.76 \mathrm{~b}-\mathrm{e}$ & $4.71 \mathrm{~b}-\mathrm{e}$ & $4.75 \mathrm{~b}-\mathrm{e}$ & $4.78 \mathrm{~b}-\mathrm{e}$ & $4.75 \mathrm{~A}$ \\
\hline 1,600 & $5.03 \mathrm{abc}$ & $4.28 \mathrm{de}$ & $4.86 \mathrm{bcd}$ & 4.74 b-e & $4.72 \mathrm{~A}$ \\
\hline 2,400 & 4.46 cde & $4.60 \mathrm{~b}-\mathrm{e}$ & $4.59 \mathrm{~b}-\mathrm{e}$ & $5.12 \mathrm{ab}$ & $4.69 \mathrm{~A}$ \\
\hline 3,200 & $4.63 \mathrm{~b}-\mathrm{e}$ & $4.27 \mathrm{e}$ & $4.86 \mathrm{bcd}$ & $4.99 \mathrm{abc}$ & $4.68 \mathrm{~A}$ \\
\hline \multirow[t]{2}{*}{ Mean } & $4.62 \mathrm{C}$ & $4.67 \mathrm{BC}$ & $4.81 \mathrm{AB}$ & $4.90 \mathrm{~A}$ & \\
\hline & Leaf $\mathrm{Ca}$ & \multicolumn{2}{|c|}{ Stem Ca } & \multicolumn{2}{|c|}{ Root $\mathrm{Ca}$} \\
\hline $\mathrm{NaCl}$ level & $* *$ & \multicolumn{2}{|c|}{$* *$} & \multicolumn{2}{|c|}{ NS } \\
\hline Zn level & $* *$ & \multicolumn{2}{|c|}{$* *$} & \multicolumn{2}{|c|}{ NS } \\
\hline $\mathrm{NaCl} \times \mathrm{Zn}$ & $* *$ & \multicolumn{2}{|c|}{$* *$} & \multicolumn{2}{|c|}{$* *$} \\
\hline
\end{tabular}

NS Non-significant

** Significant at $P \leq 0.01$.

${ }^{\dagger}$ Means followed by the same letter (small letters for means and capital letters for means of main effects) are not signi?cantly different according to Tukey's HSD test at $P \leq 0.05$.

paralleled by decreases in RGR [36]. This salt-induced growth inhibition was associated with the accumulation of salt ions $(\mathrm{Na}$ and $\mathrm{Cl})$ in plant tissues and with a nutrient imbalance. Salinity up to $1,600 \mathrm{mg} \mathrm{kg}^{-1}$ soil significantly increased $\mathrm{Ca}$ levels in leaf of pistachio seedlings, however, in stem tissues, higher salinity concentrations show significant $\mathrm{Ca}$ concentration compared with control. Salinity had no significant effect on root calcium concentration. Zinc application significantly
Table VI. Effects of zinc ( $\mathrm{Zn}$ ) treatments on the K content of leaves, stems and roots of Pistacia vera $\mathrm{L}$. cv. Badami seedlings under $\mathrm{NaCl}$ stress (DW: dry weight). Values are means of 4 replicates.

\begin{tabular}{|c|c|c|c|c|c|}
\hline \multicolumn{6}{|c|}{ Zn levels $\left(\mathrm{mg} \mathrm{kg}^{-1}\right)$} \\
\hline $\begin{array}{l}\mathrm{NaCl} \text { levels } \\
\left(\mathrm{mg} \mathrm{kg}^{-1}\right)\end{array}$ & 0 & 5 & 10 & 20 & Means \\
\hline \multicolumn{6}{|c|}{ Leaf $\mathrm{K}\left(\mathrm{mg} \mathrm{kg}^{-1} \mathrm{DW}\right)$} \\
\hline 0 & $12.40 \mathrm{def}^{\dagger}$ & $18.00 \mathrm{~b}$ & $15.50 \mathrm{bc}$ & $23.00 \mathrm{a}$ & $17.22 \mathrm{~A}$ \\
\hline 800 & $11.60 \mathrm{ef}$ & $17.50 \mathrm{~b}$ & $12.75 \mathrm{cde}$ & $12.50 \mathrm{def}$ & $13.58 \mathrm{~B}$ \\
\hline 1,600 & $6.80 \mathrm{~g}$ & $18.00 \mathrm{~b}$ & $15.50 \mathrm{bc}$ & $13.25 \mathrm{cde}$ & $13.38 \mathrm{~B}$ \\
\hline 2,400 & $13.20 \mathrm{cde}$ & $15.40 \mathrm{bc}$ & $13.00 \mathrm{cde}$ & $13.25 \mathrm{cde}$ & $13.71 \mathrm{~B}$ \\
\hline 3,200 & 14.20 cde & $14.50 \mathrm{~cd}$ & $9.80 \mathrm{f}$ & $6.00 \mathrm{~g}$ & $11.12 \mathrm{C}$ \\
\hline Mean & $11.64 \mathrm{C}$ & $16.68 \mathrm{~A}$ & $13.31 \mathrm{~B}$ & $13.60 \mathrm{~B}$ & \\
\hline \multicolumn{6}{|c|}{ Stem K $\left(\mathrm{g} \mathrm{kg}^{-1} \mathrm{DW}\right)$} \\
\hline 0 & $9.80 \mathrm{def}$ & $15.50 \mathrm{a}$ & $11.25 \mathrm{~b}-\mathrm{e}$ & $10.50 \mathrm{cde}$ & $11.76 \mathrm{~A}$ \\
\hline 800 & $10.00 \mathrm{def}$ & $13.00 \mathrm{~b}$ & $11.25 \mathrm{~b}-\mathrm{e}$ & $12.00 \mathrm{bcd}$ & $11.56 \mathrm{AB}$ \\
\hline 1,600 & $11.60 \mathrm{~b}-\mathrm{e}$ & $12.00 \mathrm{bcd}$ & $11.30 \mathrm{~b}-\mathrm{e}$ & $12.00 \mathrm{bcd}$ & $11.72 \mathrm{~A}$ \\
\hline 2,400 & $7.75 \mathrm{fg}$ & $12.40 \mathrm{bc}$ & 10.50 cde & $12.40 \mathrm{bc}$ & $10.76 \mathrm{~B}$ \\
\hline 3,200 & $9.30 \mathrm{efg}$ & $7.00 \mathrm{~g}$ & $13.00 \mathrm{~b}$ & $10.00 \mathrm{def}$ & $9.82 \mathrm{C}$ \\
\hline Mean & $9.69 \mathrm{~B}$ & $11.98 \mathrm{~A}$ & $11.46 \mathrm{~A}$ & $11.38 \mathrm{~A}$ & \\
\hline \multicolumn{6}{|c|}{ Root K $\left(\mathrm{g} \mathrm{kg}^{-1} \mathrm{DW}\right)$} \\
\hline 0 & 7.00 ef & 9.40 cde & $14.20 \mathrm{a}$ & $8.00 \mathrm{def}$ & $9.65 \mathrm{~A}$ \\
\hline 800 & 7.00 ef & $12.40 \mathrm{ab}$ & $12.00 \mathrm{abc}$ & $6.75 \mathrm{ef}$ & $9.53 \mathrm{~A}$ \\
\hline 1,600 & $7.40 \mathrm{def}$ & $10.00 \mathrm{bcd}$ & $8.00 \mathrm{def}$ & $7.75 \mathrm{def}$ & $8.28 \mathrm{~B}$ \\
\hline 2,400 & $7.60 \mathrm{def}$ & $7.80 \mathrm{def}$ & $8.00 \mathrm{def}$ & $7.50 \mathrm{def}$ & $7.72 \mathrm{~B}$ \\
\hline 3,200 & $7.60 \mathrm{def}$ & $7.50 \mathrm{def}$ & $7.75 \mathrm{def}$ & $6.25 \mathrm{f}$ & $7.27 \mathrm{~B}$ \\
\hline \multirow[t]{2}{*}{ Mean } & $7.32 \mathrm{~B}$ & $9.42 \mathrm{~A}$ & $9.99 \mathrm{~A}$ & $7.25 \mathrm{~B}$ & \\
\hline & Leaf $K$ & \multicolumn{2}{|c|}{ Stem K } & \multicolumn{2}{|c|}{ Root $\mathrm{K}$} \\
\hline $\mathrm{NaCl}$ level & $* *$ & \multicolumn{2}{|c|}{$* *$} & \multicolumn{2}{|c|}{$* *$} \\
\hline Zn level & $* *$ & \multicolumn{2}{|c|}{$* *$} & \multicolumn{2}{|c|}{$* *$} \\
\hline $\mathrm{NaCl} \times \mathrm{Zn}$ & $* *$ & \multicolumn{2}{|c|}{$* *$} & \multicolumn{2}{|c|}{$* *$} \\
\hline
\end{tabular}

** Significant at $P \leq 0.01$.

${ }^{\dagger}$ Means followed by the same letter (small letters for means and capital letters for means of main effects) are not significantly different according to Tukey's HSD test at $P \leq 0.05$.

increased the calcium concentration of leaf, stem and root tissues. At all salinity levels, Ca concentration of leaf significantly increased under $\mathrm{Zn}$ application at 5 and $10 \mathrm{mg} \mathrm{kg}^{-1}$ soil. The Ca concentration of stem and root significantly was affected by $\mathrm{Zn}$ application under salinity stress (table V). Under salinity stress, potassium concentrations of leaf, stem and root significantly decreased compared with control treatment.The $\mathrm{K}$ concentration was more pronounced in stem and leaf than in root. Increasing $\mathrm{Zn}$ concentration in soil up to $10 \mathrm{mg} \mathrm{kg}^{-1}$ soi, 
led to a significant increase in $\mathrm{K}$ concentration of leaf, stem and root tissues. Under $\mathrm{Zn}$ level of $20 \mathrm{mg} \mathrm{kg}^{-1}$ soil, the $\mathrm{K}$ concentration of root decreased (table VI). $\mathrm{NaCl}$ and $\mathrm{Zn}$ applications had no significant effect on magnesium $(\mathrm{Mg})$ concentration of leaf, stem and root of pistachio seedlings (Data not shown).

The specific absorption rates (SAR) of $\mathrm{K}^{+}, \mathrm{Ca}^{+2}$ and $\mathrm{Zn}$ were relatively high in non-saline conditions. Salinity severely reduced the SARs of these elements. Differences in SARs among $\mathrm{Zn}$ treatments were evident after 100 days of salt treatment. Zinc application increased the specific absorption rate (SAR) for these elements under salinity stress. The SAR values of ${ }^{+}$and $\mathrm{Ca}^{+2}$ were higher in 5 and $10 \mathrm{mg} \mathrm{Zn} \mathrm{kg}^{-1}$ soil treatments than in $20 \mathrm{mg} \mathrm{Zn} \mathrm{kg}{ }^{-1}$ soil treatment (figure 1). Clearly, the maximum SAR of Zn was observed in the $20 \mathrm{mg}$ $\mathrm{Zn} \mathrm{kg}^{-1}$ soil treatment. Saline inhibition on the uptake rate of $\mathrm{Zn}$ was evident in low salinity levels. In contrast to these elements, there were no significant salt and $\mathrm{Zn}$ treatment effects on the rate of $\mathrm{Mg}^{2+}$ uptake $\left(\mathrm{SAR}_{\mathrm{Mg}}\right.$ ). The SAR of salt ions ( $\mathrm{Na}$ and $\mathrm{Cl}$ ) were very low in non-saline conditions, but salinity increased the absorption rates of $\mathrm{Na}^{+}$and $\mathrm{Cl}^{-}$as ranged from 0.03 to $0.1 \mathrm{mg} \mathrm{g}^{-1}$ root day ${ }^{-1}$ and from 0.6 to $1.2 \mathrm{mg} \mathrm{g}^{-1}$ root day $^{-1}$ respectively. These corresponding values decreased to about half amount by $\mathrm{Zn}$ application (figure 1).

Specific utilization rate on leaf basis $\left(\mathrm{SUR}_{\mathrm{L}}\right)$ showed similar trends for all of the elements studied. There was a significant decrease in $\mathrm{SUR}_{\mathrm{L}}$ with increasing salinity in all $\mathrm{Zn}$ treatments. Zinc application especially in $10 \mathrm{mg} \mathrm{kg}^{-1}$ soil increased the $\mathrm{SUR}_{\mathrm{L}}$ for all elements. The $\mathrm{SUR}_{\mathrm{L}}$ for $\mathrm{Mg}^{+2}$ was higher than $\mathrm{K}^{+}$and $\mathrm{Ca}^{+2}$, and the highest $\mathrm{SUR}_{\mathrm{L}}$ was for $\mathrm{Zn}$. Zinc application showed a significant decrease in the utilization rate of $\mathrm{Na}^{+}$and $\mathrm{Cl}^{-}$, however under salinity stress higher $\mathrm{SU}_{\mathrm{RL}}$ for the other nutrients were sustained by $\mathrm{Zn}$ treatments. For all elements, the 5- and 10-mg Zn kg-1 soil treatments exhibited higher $\mathrm{SUR}_{\mathrm{L}}$ values than the $20 \mathrm{mg} \mathrm{Zn} \mathrm{kg}{ }^{-1}$ soil treatment (figure 2).

Regression equations of relative growth rate (RGR) with SAR and with SUR $\mathrm{L}_{\mathrm{L}}$ of all elements studied were calculated to evaluate the relative importance of these parameters for each nutrient with respect to their effects on RGR. The correlation coefficients are presented in table VII. The RGR was significantly correlated with the SARs of $\mathrm{K}$ and Ca. No significant correlation was observed for $\mathrm{Mg}$ and $\mathrm{Zn}$. The SARs of salt ions ( $\mathrm{Na}$ and $\mathrm{Cl}$ ) had a significant negative correlation with RGR. Relative growth rate also showed a significant correlation with the SURs of all elements studied (table VII).

The results emphasize the lower absorption rates of $\mathrm{K}$, $\mathrm{Ca}$ and $\mathrm{Zn}$ (figure 1) and the lower utilization rates of $\mathrm{K}$, $\mathrm{Ca}, \mathrm{Zn}$ and $\mathrm{Mg}$ (figure 2). Similar findings were achieved in annual sweet clover (Melilotus officinalis) and citrus rootstocks [24,37]. Similarly, Alpaslan et al. [2] found that a sufficient $\mathrm{Zn}$ supply could reduce $\mathrm{Na}$ and $\mathrm{Cl}$ accumulation and contribute to salt tolerance in tomato plants. Elevated $\mathrm{K}$ and Ca contents in roots and stems of lettuce [38] and pepper [39] supplied with $\mathrm{Zn}$ at saline conditions is confirmed. In $\mathrm{Zn}$ deficient plants, the loss of membrane integrity and the increase in membrane permeability are very common in different plant species [16]. The loss of membrane integrity under $\mathrm{Zn}$ deficiency may affect the uptake and accumulation of $\mathrm{Na}$ and
Table VII. Correlation coefficients of the regression equations for the relative growth rates (RGR) with the specific absorption rates (SAR) and the utilization rates on a leaf basis $\left(\mathrm{SUR}_{\mathrm{L}}\right)$ of mineral elements in Pistacia vera L. cv. Badami seedlings under $\mathrm{NaCl}$ stress and $\mathrm{Zn}$ treatments.

\begin{tabular}{ccc}
\hline Elements & & \\
\hline & $\mathrm{SAR}$ & $\mathrm{SUR}_{\mathrm{L}}$ \\
$\mathrm{Na}$ & $-0.90^{* *}$ & $0.76^{* *}$ \\
$\mathrm{Cl}$ & $-0.83^{* *}$ & $0.73^{* *}$ \\
$\mathrm{~K}$ & $0.92 * *$ & $0.81^{* *}$ \\
$\mathrm{Ca}$ & $0.77 * *$ & $0.86^{* *}$ \\
$\mathrm{Mg}$ & $0.44 \mathrm{NS}$ & $0.87 * *$ \\
$\mathrm{Zn}$ & $0.39 \mathrm{NS}$ & $0.88^{* *}$ \\
\hline
\end{tabular}

NS Non-significant.

** Significant at $P \leq 0.01$.

$\mathrm{Cl}$ at toxic levels in plants. Previously, Norvell and Welch [4] reported that adequate supply of $\mathrm{Zn}$ is important in controlling root uptake and stem accumulation of $\mathrm{Na}$ and $\mathrm{Cl}$. The other role of $\mathrm{Zn}$ is its function as inhibitor on the anion/ $\mathrm{Cl}$ channels. Zn, acting as an inhibitor on hyperpolarization-activated inward anion/ $\mathrm{Cl}$ channels, may be beneficial for reducing the $\mathrm{Cl}$ absorption and enhancing the $\mathrm{NO}_{3}$ uptake to the pistachio seedlings exposed to salt stress. This role of $\mathrm{Zn}$ has also been reported by other researchers [6].

Unfavorable environmental factors such as salinity lead to sharp changes in the balance of phytohormones associated with not only the accumulation of ABA, but also with a decline in the level of the growth activating hormones IAA and cytokinins [6]. Zinc might be effective in alleviating salt stress damage on plant growth via maintaining hormones balance within plant tissues. In the current study, increasing salinity resulted in a significant accumulation of ABA, a progressive decline in IAA and a significant decrease in the level of cytokinins. Treatment with Zn prevented the salinity-induced decline in concentration of IAA and cytokinins in seedlings and reduced the accumulation of ABA (figures $3 A-3 C$ ). Zinc deficiency reduced the IAA and cytokinins regardless of $\mathrm{NaCl}$ levels. As Zn levels increased, IAA and cytokinins concentrations significantly developed (figures $3 A$ and $3 C$ ). Zinc at the concentration of $20 \mathrm{mg} \mathrm{kg}^{-1}$ soil significantly increased cytokinins compared to the control, but its effect was smaller than 5 and/or $10 \mathrm{mg} \mathrm{Zn} \mathrm{kg}{ }^{-1}$ soil. The reverse trend was observed in the case of ABA. Abscisic acid level of the $\mathrm{Zn}$ deficient seedlings was higher than those supplied with $\mathrm{Zn}$, particularly at higher $\mathrm{NaCl}$ levels. Seedlings supplied with $10 \mathrm{mg} \mathrm{Zn} \mathrm{kg}^{-1}$ maintained ABA of approximately $272.92 \mathrm{ng} \mathrm{g}^{-1} \mathrm{FW}$, followed a slight rise with 5 and $20 \mathrm{mg} \mathrm{Zn} \mathrm{kg}^{-1}$ soil (figure $3 B$ ).

Under salt stress an enhancement of IAA and CKs by decreasing $\mathrm{ABA}$ concentration in the experimental pistachio seedlings were observed after $\mathrm{Zn}$ amendment. In line with this result, such a pattern is well documented for IAA in bean (Phaseolus vulgaris) [17], cytokinins in lupin (Lupinus albus) [41] and ABA in Ricinus communis and Xanthium strumarium [42]. Cakmak et al. [17] confirmed the role of $\mathrm{Zn}$ in protein synthesis and demonstrated that the decrease in IAA level in Zn-deficient plants, also that in Zn-deficient plants the 

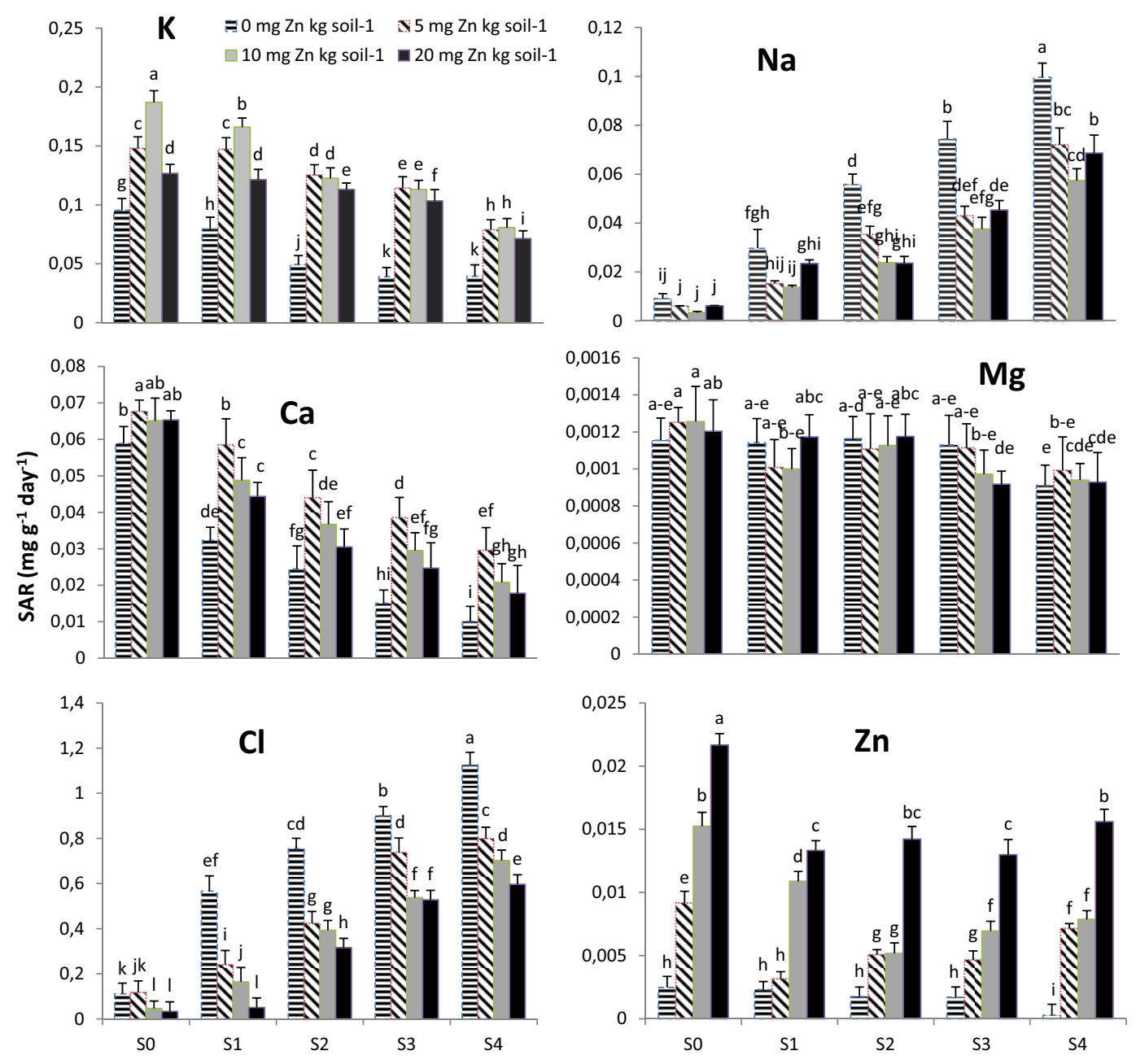

Figure 1. The effects of soil application of zinc ( $\mathrm{Zn}$ ) on specific absorption rates (SAR) of $\mathrm{K}, \mathrm{Na}, \mathrm{Ca}, \mathrm{Mg}, \mathrm{Cl}$ and $\mathrm{Zn}$ in seedlings of Pistacia vera L. cv. Badami under $\mathrm{NaCl}$ stress. Bars represent means and error bars represent standard error $(n=4)$. Bars having different letters are significantly different at the $5 \%$ level by Tukey HSD. S0, S1, S2, S3 and S4 refer to 0, 800, 1,600, 2,400, and 3,200 mg NaCl kg-1 soil, respectively.

conversion of tryptophan to IAA specifically inhibited by enhanced oxidation. Zn deficiency and/or saline conditions induced oxidative depredation of IAA and cytokinins $[43,44]$ Iron, by catalyzing the Haber-Weiss reaction, is responsible for the $\mathrm{OH}^{\bullet}$ production. IAA is extremely sensitive to $\mathrm{OH}^{\bullet}$, and can be oxidized rapidly upon exposure to high concentrations of $\mathrm{OH}^{\bullet}[43,44]$. Besides nonenzymic oxidation, IAA and also cytokinins are also oxidized by $\mathrm{H}_{2} \mathrm{O}_{2}$ dependent peroxidases $[45,46]$, and $\mathrm{O}_{2}^{\bullet}$ and ${ }^{1} \mathrm{O}_{2}$ are involved in this degradation pathway [47]. Higher peroxidase activity, $\mathrm{O}_{2}^{\bullet}$ in $\mathrm{Zn}$ deficient plants under salinity stress may result in enhanced oxidative degradation of IAA and cytokinins. ROS-scavenging antioxidant enzymes, such as superoxide dismutase (SOD), catalase (CAT), and ascorbate peroxidase (APX), play a vital role in removing these destructive oxidant species. The positive effects of $\mathrm{Zn}$ on antioxidant enzyme activity scavenging the reactive oxygen species (ROS) produced in response to salt stress and/or Zn deficiency are well demonstrated [16]. Tavallali et al. [48] reported that pistachio seedlings treated with Zn had very high SOD, CAT and APX activity, indicating that there was efficient ROS scavenging activity in the system. This could be another relevant reason why $\mathrm{Zn}$ deficient plants become very sensitive to ROS when grown under saline conditions.

Osmolytes accumulation is one of the most important responses of cell structures to environmental stress and could be an adaptive mechanism in this condition [49]. In this study, proline significantly increased in leaves of salt stressed plants compared to the control treatment. Regardless of salt concentration, proline was significantly reduced by $\mathrm{Zn}$ addition. Interaction of $10 \mathrm{mg} \mathrm{Zn} \mathrm{kg}^{-1}$ regardless of $\mathrm{NaCl}$ levels caused a significant decrease in proline as compared with other $\mathrm{Zn}$ levels (figure 4A). Thus, increased proline level in this experiment could be considered as a response to stress induction which was decreased by $\mathrm{Zn}$ (figure $4 A$ ). In line with this result, Saleh and Maftoon [50] found that increased $\mathrm{Zn}$ concentration combined with salinity stress leads to a reduction in proline accumulation in rice. The interaction between soil salinity 

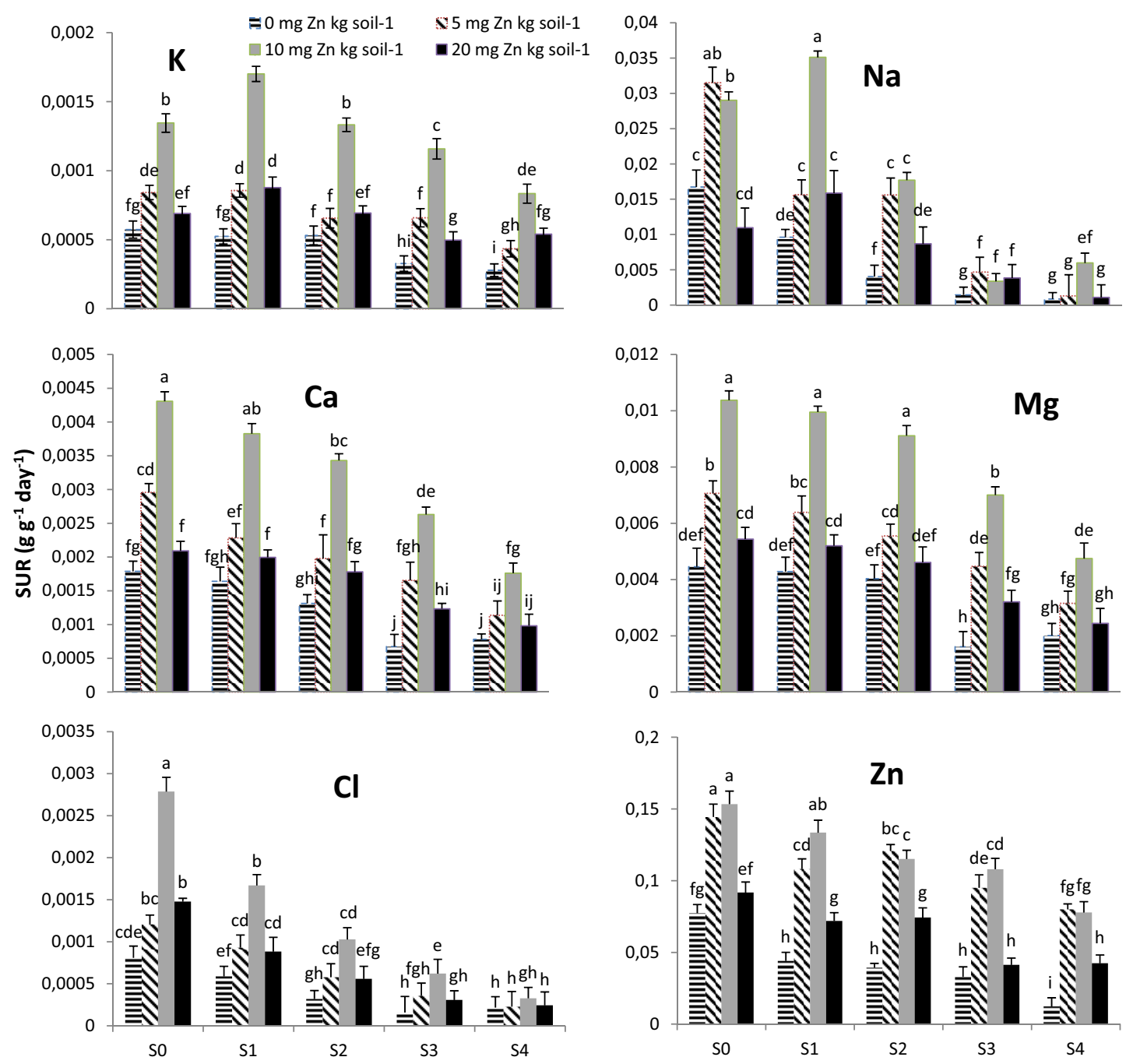

Figure 2. The effects of soil application of zinc ( $\mathrm{Zn}$ ) on specific utilization rates (SUR) of $\mathrm{K}, \mathrm{Na}, \mathrm{Ca}, \mathrm{Mg}, \mathrm{Cl}$ and $\mathrm{Zn}$ in seedling leaves of pistachio 'Badami' under $\mathrm{NaCl}$ stress. Bars represent means and error bars represent standard error $(n=4)$. Bars having different letters are significantly different at the $5 \%$ level by Tukey HSD. S0, S1, S2, S3 and S4 refer to 0, 800, 1,600, 2,400, and 3,200 mg NaCl kg ${ }^{-1}$ soil, respectively.

and $\mathrm{Zn}$ application resulted in a marked decrease in proline content in Salvia officinalis, which also has been observed by Hendawy and Khalid [51]. It seems that this may be a result of this fact that proline accumulation in plant is mainly known as a symptom of stress damage, not as a salinity resistance indicator [52]. Under stress conditions, the great amount of proline forms during the oxidation of carbohydrates and proteins [53]. Cakmak [16] suggested $\mathrm{Zn}$ as an excellent protective antioxidant may participate in proline production due to its role in alleviating stress.

Increasing $\mathrm{NaCl}$ without $\mathrm{Zn}$ significantly enhanced the concentration of other osmolytes, choline and glycine betaine compared with the control (figures $4 B$ and $4 C$ ). The interaction between soil salinity and $\mathrm{Zn}$ application especially at $20 \mathrm{mg} \mathrm{kg}^{-1}$ soil resulted in a significant decrease in choline contents in comparison with no $\mathrm{Zn}$ treatment. Choline contents were the lowest at $20 \mathrm{mg} \mathrm{Zn} \mathrm{kg}^{-1}$ soil (figure 4B). Zinc application without $\mathrm{NaCl}$ significantly increased the glycine betaine contents compared with control, however no significant differences were observed among $\mathrm{Zn}$ levels (figure $4 C$ ). A decrease in the proline and choline contents in leaves under non-saline condition amended with 10 and $20 \mathrm{mg} \mathrm{Zn} \mathrm{kg}^{-1}$ soil were considerably lower than those under control and/or $5 \mathrm{mg} \mathrm{Zn} \mathrm{kg}{ }^{-1}$ soil (figures $4 A$ and $4 B$ ).

Glycine-betaine (GB) is a quaternary nitrogenous compound which plays an important role in osmoregulation in various organisms and plants under salinity stress [54]. Glycinebetaine is synthesized from choline through two steps:

$$
\text { Choline } \rightarrow \text { betainealdehyde } \rightarrow \text { glycinebetaine [55] }
$$

The activity of GB synthesizing enzymes (e.g., betaine aldehydehydrogenase) increased in several plant species at high $\mathrm{NaCl}$ concentration [56]. Exogenous application of GB enhanced the growth of plants under different stresses [57]. The addition of choline, GB precursor, to the growth medium increases acclimation of salt sensitive wheat genotype to $\mathrm{NaCl}$ stress [58]. 

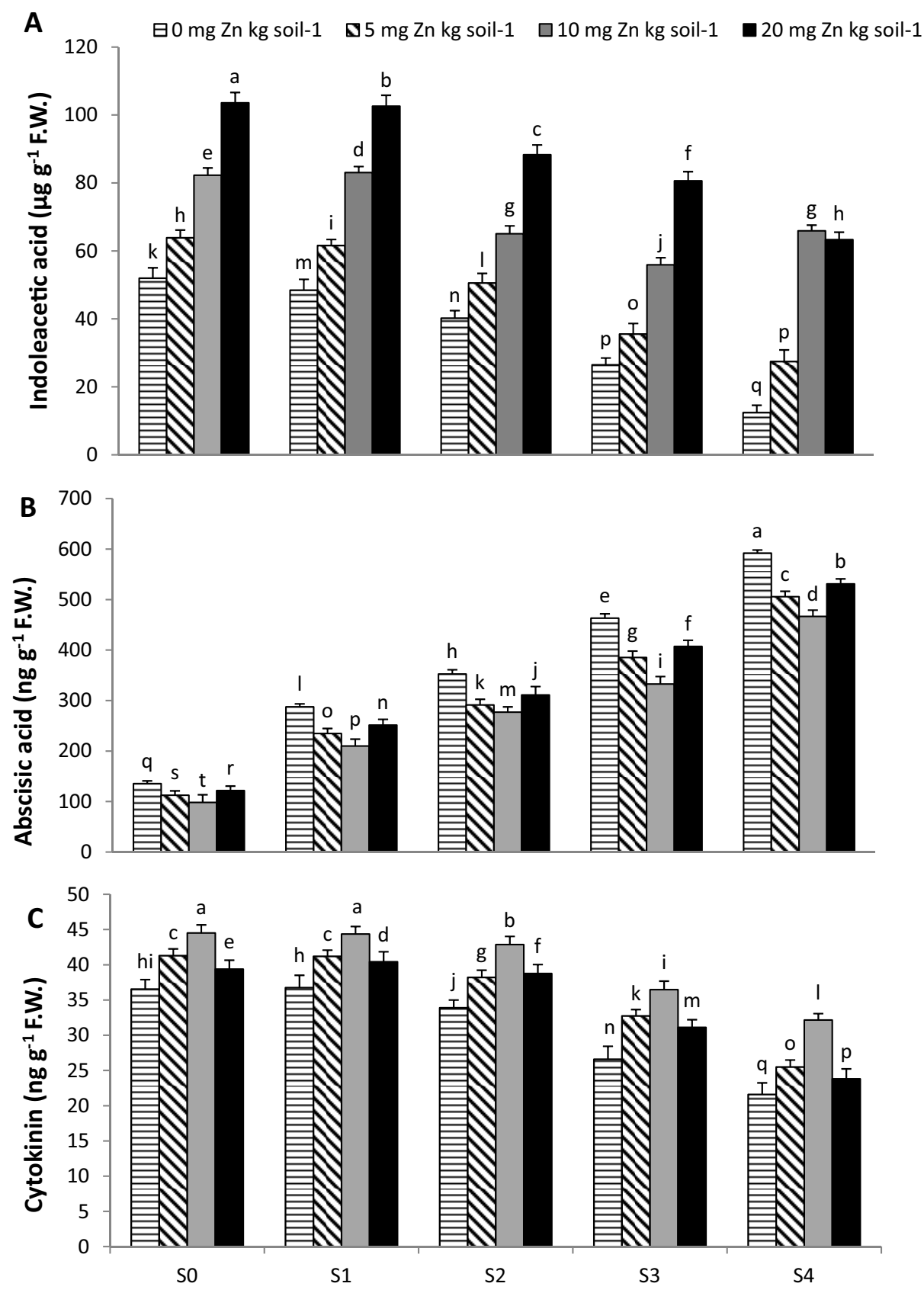

Figure 3. The effects of soil application of zinc $(\mathrm{Zn})$ on indoleacetic acid (IAA) (A), abscisic acid (ABA) (B) and cytokinin (C) contents in seedling leaves of pistachio 'Badami' under $\mathrm{NaCl}$ stress. Bars represent means and error bars represent standard error $(n=4)$. Bars having different letters are significantly different at the 5\% level by Tukey HSD. S0, S1, S2, S3 and S4 refer to 0, 800, 1,600, 2,400, and 3,200 mg $\mathrm{NaCl} \mathrm{kg}{ }^{-1}$ soil, respectively (F.W.: fresh weight).

Glycine betaine is mainly localized in chloroplasts [55] and its accumulation contributes to chloroplast adjustment and protection of thylakoid membrane thus maintaining photosynthetic activity [59]. Glycine betaine application increased net photosynthetic rate of salt stressed tomato and turnip which was due to increased stomatal conductance and decreased photorespiration [60]. A Zn-enhancement of GB is very beneficial for plants in order to provide osmotic adjustment and participate in stomatal regulation and facilitate the photosynthetic activity [57].

\section{Conclusion}

This study demonstrated that a $\mathrm{Zn}$ deficiency reduces the nutrient uptake and utilization and the phytohormone balance especially under saline conditions. Adequate $\mathrm{Zn}$ treatments may improve salt tolerance of Pistacia. vera 'Badami' seedlings by enhancing the accumulation of osmolytes in plant tissues and improving supplementation on $\mathrm{K}, \mathrm{Ca}$ and $\mathrm{Zn}$ absorption and utilizing efficiency. Adequate $\mathrm{Zn}$ supply was suggested to limit uptake and accumulation of $\mathrm{Na}$ in leaf and stem. 

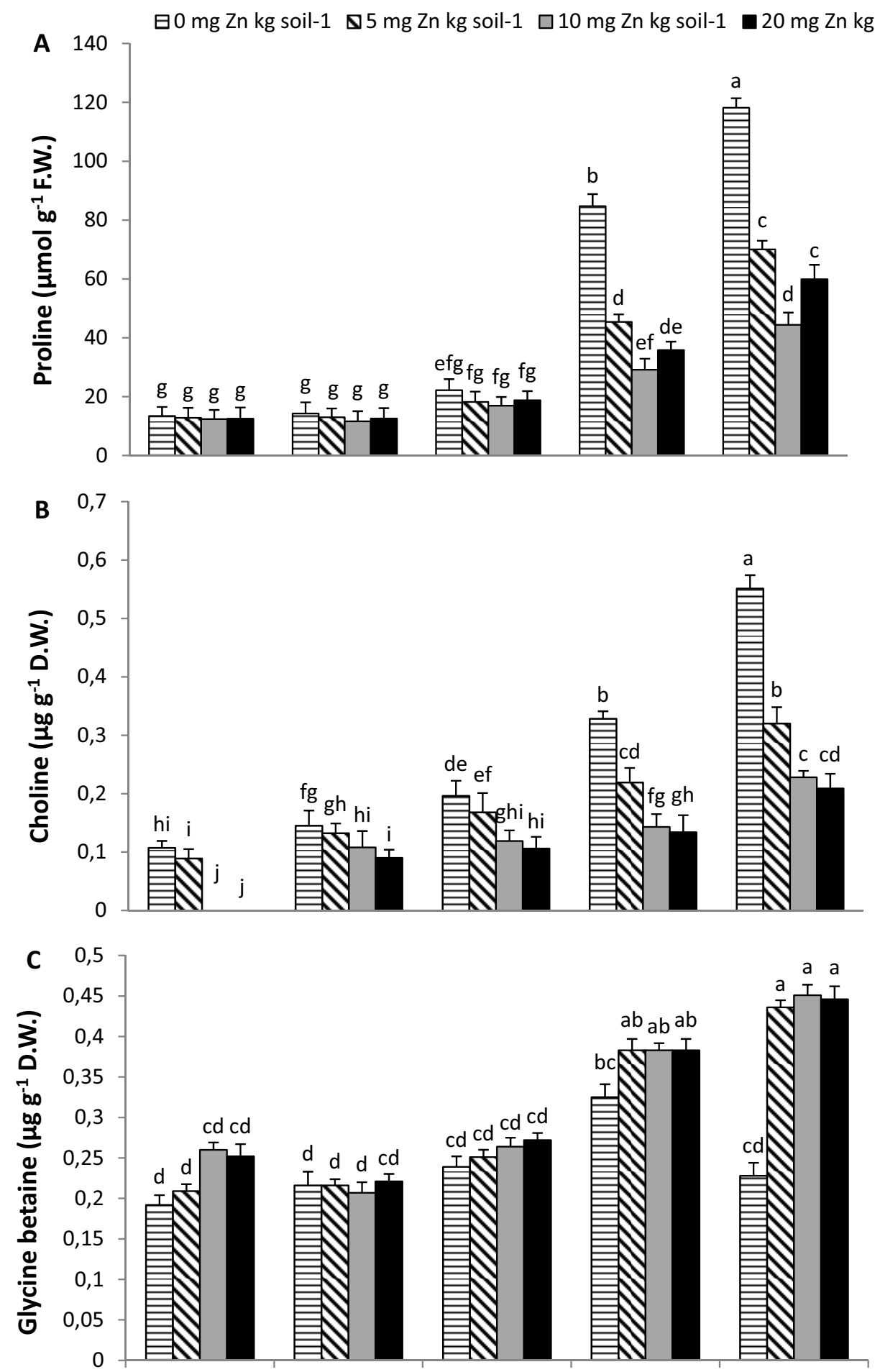

Figure 4. Effects of $\mathrm{Zn}$ on proline (A), choline (B) and glycine betaine (C) contents in seedling leaves of pistachio 'Badami' under NaCl stress. Bars represent means and error bars represent standard error $(n=4)$. Bars having different letters are significantly different at the $5 \%$ level by Tukey HSD. S0, S1, S2, S3 and S4 refer to 0, 800, 1,600, 2,400, and 3,200 $\mathrm{mg} \mathrm{NaCl} \mathrm{kg}^{-1}$ soil, respectively (F.W./D.W.: fresh/dry weight). 
The addition of $\mathrm{Zn}$ may enhance pistachio salt tolerance by reducing ABA level and enhancing IAA and cytokinin concentrations in the plants. Therefore, the application of $10 \mathrm{mg} \mathrm{Zn}$ $\mathrm{kg}^{-1}$ soil is recommended for alleviating salt-induced damages on pistachio seedlings.

\section{References}

[1] Zhu J.K., Plant salt tolerance, Trends Plant Sci. 6 (2001) 66-71.

[2] Shilpim M., Narendra T., Cold, salinity and drought stresses: an overview, Arch. Biochem. Biophys. 444 (2005) 139-158.

[3] Habibi Gh., Norouzi F., Hajiboland R., Silicon alleviates salt stress in pistachio plants, Prog. Biol. Sci. 4 (2014) 189-202.

[4] Khan M.I.R., Iqbal N., Masood A., Khan N.A., Variation in salt tolerance of wheat cultivars: role of glycine betaine and ethylene Pedosphere 22 (2012) 746-754.

[5] Jackson M., Hormones from roots as signal for the stems of stressed plants, Trends Plant Sci. 2 (1997) 22-28.

[6] Shahriaripour R., Tajabadi pour A., Mozaffari V., Dashti H., Adhami E., Effects of salinity and soil zinc application on growth and chemical composition of pistachio seedlings, J. Plant Nutr. 33(8) (2010) 1166-1179.

[7] Razavi nasab A., Tajabadi pour A., Shirani H., Effect of salinity and nitrogen application on growth, chemical composition and some biochemical indices of pistachio seedlings (Pistacia vera L.), J. Plant Nutr. 37(10) (2014) 1612-1626.

[8] Sepaskhah A.R., Maftoun M., Growth and chemical composition of pistachio seedlings as influenced by irrigation regimes and salinity levels of irrigation water: I. Growth. J. Am. Soc. Hort. Sci. 57 (1981) 469-476.

[9] Grattan S.R., Grieve C.M., Salinity-mineral nutrient relations in horticultural crops, Sci. Hort. 78 (1999) 127-157.

[10] Behboudian M.H., Walker R.R., Torokfaivy E., Effect of water stress and salinity on photosynthesis of pistachio, Sci. Hort. 29 (1986) 251-261.

[11] Picchioni G.A., Miyamota S., Salt effects on growth and ion uptake of pistachio rootstock seedlings, J. Am. Soc. Hort. Sci. 115 (1990) 647-653.

[12] Ferguson L., Poss J.A., Grattan S.R., Grieve C.M., Wang D., Wilson C., Donovan Chao C.T., Pistachio rootstocks influence scion growth and ion relations under salinity and boron stress, J. Am. Soc. Hort. Sci. 127 (2002) 194-199.

[13] Ranjbar A., Damme P.van., Samson R., Lemeur R., Leaf water status and photosynthetic gas exchange of Pistacia khinjuk and P. mutica exposed to osmotic drought stress, Acta Hort. 591 (2002) 423-428.

[14] Aravind P., Prasad M.N.V., Zinc protects chloroplasts and associated photochemical functions in cadmium exposed Ceratophyllum demersum L., a fresh water macrophyte, Plant Sci. 166 (2004) 1321-1327.

[15] Welch R.M., The impact of mineral nutrient in food crops on global human health, Plant Soil 247 (2002) 83-90.

[16] Cakmak I., Possible roles of zinc in protecting plant cells from damage by reactive oxygen species, New Phytol. 146 (2000) 185-205.
[17] Cakmak I., Marschner H., Bangerth F,. Effect of zinc nutritional status on growth, protein metabolism and levels of indole-3acetic acid and other phytohormones in bean (Phaseolus vulgaris L.), J. Exp. Bot. 40 (1989) 405-412.

[18] Marschner H., Cakmak I., High light intensity enhances chlorosis and necrosis in leaves of zinc potassium, and magnesium deficient bean (Phaseolus vulgaris L.) plant, J. Plant Physiol. 134 (1989) 308-315.

[19] Welch R.M., Webb M.J., Lonegaran J.E., Zinc in membrane function and its role in phosphorus toxicity. in: Scaife A. (Ed.), Proceedings of the $9^{\text {th }}$ International Plant Nutrition. Coll. (ed. A. Scaife), Commonw. Agric. Bur., Farnham Royal. Bucks, UK, 1982 pp. 710-715.

[20] Alpaslan M, Inal A., Gunes A., Cikili Y., Oscan H., Effect of zinc treatment on the alleviation of sodium and chloride injury in tomato [(Lycopersicum esculentum L.) Mill. Cv. Late] grown under salinity, Turk. J. Bot 23 (1999) 1-6.

[21] Eskandari E., Mozaffari V., Tajabadi Pour A., Effect of salinity and copper on growth and chemical composition of pistachio seedlings, J. Plant Nut. 37 (2014) 1063-1079.

[22] Tandon H.L.S., Methods of Analysis of Soils, Plants, Waters and Fertilizers, Fertilizer Development and Consultation Organisation, New Delhi, India, 1998.

[23] Hunt R., Plant growth curves. An introduction to the functional approach to plant growth analysis, Edward Arnold, London, 1982.

[24] Romero J.M., Maran T., Murillo J.M., Long-term responses of Melilotus segetalis to salinity. II. Nutrient absorption and utilization, Plant Cell Environ. 17 (1994) 1249-1255.

[25] Novakova M., Motyka V., Dobrev P.I., Malbeck J., Gaudinova A., Vankova R., Diurnal variation of cytokinin, auxin and abscisic acid levels in tobacco leaves, J. Exp. Bot. 56 (2005) 2872883

[26] Dobrev P.I., Havlicek L., Vagner M., Malbeck J., Kaminek M., Puriflcation and determination of plant hormones auxin and abscisic acid using solid phase extraction and two-dimensional high performance liquid chromatography, J. Chromato. 1075 (2005) 159-166.

[27] Lexa M., Genkov T., Malbeck J., Machackova I., Brzohohaty B., 2003. Dynamics of endogenous cytokinins pool in tobacco seedlings: a modelling approach, Ann. Bot. 91 (2003) 585-597.

[28] Bates L.S., Waldeen R.P., Teare I.D., Rapid Determination of Free Proline for Water-stressed Studies, Plant Soil 39 (1973) 205-207.

[29] Grieve C.M., Grattan S.R., Rapid assay for the determination of water soluble quaternary ammonium compounds, Plant Soil 70 (1983) 303-307.

[30] Uriu K., Pearson J., Diagnosis and correction of nutritional problems. Calif, Pistachio Ind. Ann. Rep. 1981.

[31] Uriu K., Pearson J., Diagnosis and correction of nutritional problems, including the crinkle leaf disorder, Calif. Pistachio Ind. Ann. Rep. 1983.

[32] Gunes A., Inal A., Alpaslan M., Effect of salinity on stomatal resistance, proline and mineral composition of pepper, J. Plant Nutr. 19 (1996) 389-396. 
[33] Jamalomidi M., Esfahani M., Carapetian J., Zinc and salinity interaction on agronomical traits, chlorophyll and proline content in lowland rice (Oryza sativa L.) genotypes, Pak. J. Biol. Sci. 9 (2006) 1315-1319.

[34] Khoshgoftarmanesh A.H., Shariatmadari H., Karimian N., Khajehpour M.R., Responses of wheat genotypes to zinc fertilization under saline soil conditions, J. Plant Nutr. 29 (2006) 1543-1556.

[35] Genc Y., McDonald G.K., Graham R.D., The interactive effects of zinc and salt on growth of wheat. in: Li C.J. (Ed.), Plant Nutrition for Food Security, Human Health and Environmental Protection, Tsinghua University Press, Beijing, China, 2005.

[36] Pardia A.K., Das A.B., Salt tolerance and salinity effects on plants: a review, Ecotoxicol. Environ. Saf. 60 (2005) 324-349.

[37] Ruiz D., Martinez V., Cerda A., Citrus response to salinity: growth and nutrient uptake, Tree Physiol. 17 (1997) 14-150.

[38] Mohammadi P., Khoshgoftarmanesh A.H., The effectiveness of synthetic zinc( $\mathrm{Zn}$ )-amino chelates in supplying $\mathrm{Zn}$ and alleviating salt-induced damages on hydroponically grown lettuce, Sci. Hort. 172 (2014) 117-123.

[39] Aktas H., Abak K., Cakmak I., Genotypic variation in the response of pepper to salinity, Sci. Hort. 110 (2006) 260-266.

[40] Norvell W.A., Welch R.M., Growth and nutrient uptake by barley (Hordeum vulgare L. cv. Herta): studies using an N(2-hydroxyethyl)ethylenedinitrilotriacetic acid-buffered nutrient solution technique. I. Zinc ion requirements, Plant Physiol. 101 (1993) 619-625.

[41] Davey Z.E., Van Staden J., Cytokinin activity in Lupinus albus. I. Distribution in vegetative and flowering plants. Physiol, Plant. 43 (1978) 77-81.

[42] Zeevaart J.A.D., Boyer G.L., Accumulation and transport of abscisic acid and its metabolites in Ricinus and Xanthium Plant Physiol. 74 (1984) 934-939.

[43] Cakmak I., Morphologische und physiologische Veranderungen bei Zink mangelpflanzen, University Hohenheim, Ph.D. thesis, 1988.

[44] Dunlop J.R., Robacker K.K., Nutrient salts promote lightinduced degradation of indole-3-acetic acid in tissue culture media, Plant Physiol. 88 (1988) 379-382.

[45] Schneider E.A., Wightman F., 1974. Metabolism of auxin in higher plants, Ann. Rev. Plant Physiol. 25 (1974) 487-513.

[46] Gazaryan I.G., Lagrimini L.M., Ashby G.A., Thorneley N.F., Mechanism of indole-3-acetic acid oxidation by plant peroxidases: anaerobic stopped-flow spectrophotometric studies on horseradish and tobacco peroxidases, Biochem. J. 313 (1996) 841-847
[47] Metodiewa D, De Melo, M.P., Escobar J.A., Cilento G., Dunford H.B., Horseradish peroxidase-catalysed aerobic oxidation and peroxidation of indole-3-acetic acid. I. Optical spectra, Arch. Biochem. Biophys. 296 (1992) 27-33.

[48] Tavallali V., Rahemi M., Eshghi S., Kholdebarin B., Ramezanian A., Zinc alleviates salt stress and increases antioxidant enzyme activity in the leaves of pistachio (Pistacia vera L. 'Badami') seedlings. Turk. J. Agri. For. 34 (2010) 349-359.

[49] Gilbert A.G., Gadush M.V., Wilson C., Madore M.A., Amino acid accumulation in sink and source tissues of Coleus blumei Benth, during salinity stress. J. Exp. Bot. 49 (1998) 107-114.

[50] Saleh J., Maftoon M., 2008. Interactive effects of $\mathrm{NaCl}$ levels and zinc sources and levels on the growth and mineral composition of rice, J. Agric. Sci. Technol. 10 (2008) 325-336.

[51] Hendawy S.F., Khalid Kh.A., Response of sage (Salvia officinalis L.) plants to zinc application under different salinity levels, J. Appl. Sci. Res. 1 (2005) 14-155.

[52] Luttus S., Majerus V., Konet J.M., $\mathrm{NaCl}$ effects on proline metabolism in rice, Physiol. Plant. 105 (1999) 450-458.

[53] Palfi G., Effects of kinetin, 2-4-D and antimetabolites on the changes in amino acid content of withering leaves, Planta 78 (1968) 196-199.

[54] Sulpice R., Tsukaya H., Nonaka H., Mustardy L., Chen T.H.H., Murata N., Enhanced formation of flowers in salt-stressed Arabidopsis after genetic engineering of the synthesis of glycine betaine, Plant J. 36 (2003) 165-176.

[55] Rhodes D., Hanson A.D., Quaternary ammonium and tertiary sulfonium compounds in higher plants, Ann. Rev. Plant Physiol. Plant Molec. Biol 44 (1993) 357-384.

[56] McCue R.F., Hanson A.D., Drought and salt tolerance: toward understanding and application, Tibtech. 8 (1990) 35-362.

[57] Mansour M.M.F., Nitrogen containing compounds and adaptation of plants to salinity stress, Biol. Plant. 43 (2000) 491-500.

[58] Mansour M.M.F., Stadelmann E.J., Lee-Stadelmann O.Y., Salt acclimation of Triticum by choline chloride: plant growth, mineral content and cell permeability, Plant Physiol. Biochem. 31 (1993) 34-348.

[59] Genard H., Le Saos J., Hillard J., Tremolieres A., Boucaud J., Effect of salinity on lipid composition, glycinebetaine content and photosynthesis activity in chloroplasts of Suaeda maritime, Plant Physiol. 29 (1991) 421-427.

[60] Maleka P., Kontturi M., Pehu B., Somersalo S., Photosynthesis response of drought and salt-stressed tomato and turnip plants to foliar-applied glycinebetaine, Physiol. Plant. 105 (1999) 4-50.

Cite this article as: Vahid Tavallali. The effectiveness of zinc in alleviating salinity stress on pistachio seedlings. Fruits 71 (2016) $433-445$. 